\title{
Male orthopaedic surgeons and anaesthetists: equally good at estimating fluid volumes (and changing light bulbs) but equally poor at estimating procedure duration
}

Weiliang $\underline{\text { Chua }}^{1}$, MBBs, Chee Hoe Kong ${ }^{1}$, MBBS, Diarmuid Paul Murphy ${ }^{1}$, FRCS

INTRODUCTION How many orthopods does it take to change a light bulb? One - to refer to the medics for 'Darkness ?Cause'. Additionally, anaesthetists and surgeons often disagree on the estimated blood loss during surgery and the estimated procedure duration. We designed this study to compare the ability of orthopaedic surgeons and anaesthetists in: (a) estimating fluid volumes; (b) estimating procedure durations; and (c) changing light bulbs.

METHODS Participants had to either be a specialist in anaesthesia or orthopaedic surgery, or a trainee in that specialty for at least two years. Three different fluid specimens were used for volume estimation ( $44 \mathrm{~mL}, 88 \mathrm{~mL}$ and $144 \mathrm{~mL}$ ). Two videos of different lengths (140 seconds and 170 seconds), showing the suturing of a banana skin, were used for procedure duration estimation. To determine the ability at changing light bulbs, the participants had to match eight different light sockets to their respective bulbs.

RESULTS 30 male anaesthetists and trainees and 31 male orthopaedic surgeons and trainees participated in this study. Orthopaedic surgeons underestimated the three fluid volumes by $3.9 \%$ and anaesthetists overestimated by $5.1 \%(p=0.925)$. Anaesthetists and orthopaedic surgeons overestimated the duration of the two procedures by $21.2 \%$ and $43.1 \%$, respectively $(p=0.006)$. Anaesthetists had a faster mean time in changing light bulbs (70.1 seconds vs. 74.1 seconds, $p=0.319)$. CONCLUSION In an experimental environment, male orthopaedic surgeons are as good as male anaesthetists in estimating fluid volumes (in commonly seen surgical specimens) and in changing light bulbs. Both groups are poor at estimating procedure durations.

Keywords: anaesthetists, fluid estimation, light bulbs, orthopaedic surgeons, procedure duration

\section{INTRODUCTION}

Question: How many orthopaedic surgeons does it take to change a light bulb?

Answer: One; to refer to the medics for 'Darkness ?Cause'.

Jokes about the intelligence of orthopaedic surgeons are common..$^{(1)}$ In 2011, Subramaniam et al proved that orthopaedic surgeons are as intelligent and twice as strong as anaesthetists, ${ }^{(2)}$ resulting in our anaesthesia colleagues feeling that the comparison was unjust. It is not uncommon for colleagues from these two intimately related specialties to disagree on the estimated blood loss during surgery and estimated procedure duration. Often, we see anaesthetists rolling their eyes upon hearing the orthopaedic surgeon's underestimation of blood loss during surgery and attempts at over-listing cases in the elective theatre (which affects the anaesthetists' ability to leave the hospital at $5 \mathrm{pm}$ ).

Based on the Surgical Safety Checklist published by the World Health Organization, the ability of the surgeon to accurately estimate the amount of blood loss before the start of surgery is important. ${ }^{(3)}$ Good estimation of blood loss can allow rapid and adequate fluid and blood product resuscitation of the surgical patient. ${ }^{(4)}$ Good estimation of the time taken to complete a surgery is also important as it allows anaesthetists to better titrate anaesthetic drugs and enables a smoother overall workflow for the operating list. In an attempt to settle the 'dispute' between orthopaedic surgeons and anaesthetists, the present study was designed to indirectly assess the ability of orthopaedic surgeons and anaesthetists to estimate fluid volumes, estimate procedure duration and change light bulbs.

\section{METHODS}

This study was a prospective, multicentre, comparative study. Participants were recruited from three hospitals over a one-month period. To be eligible for inclusion in this study, participants had to be either: (a) a specialist in anaesthesia or orthopaedic surgery; or (b) a trainee in either of the two aforementioned specialties, with a minimum of two years' experience in that specialty. The three hospitals involved were visited on random days and the tests were conducted in the operating room pantry or common areas. All orthopaedic surgeons and anaesthetists who passed by these areas on those days were invited to participate. Only male participants were included in this study, due to the lack of eligible female participants.

As an indirect method of testing the participants' ability to estimate surgical fluid loss, we prepared three different watertight bags with commonly used surgical materials soaked with random volumes of fluid - (a) two pieces of surgical gauze swabs (4 inches $\times 4$ inches) soaked with $44 \mathrm{~mL}$ of pink chlorhexidine; (b) one piece of surgical sponge towel (12 inches $\times 12$ inches) soaked with $144 \mathrm{~mL}$ of pink chlorhexidine; and (c) a catheter

${ }^{1}$ Department of Orthopaedic Surgery, National University Hospital, Singapore

Correspondence: Dr Chua Wei Liang, Resident, Department of Orthopaedic Surgery, National University Hospital, 5 Lower Kent Ridge Road, Singapore 119074. wei_liang_chua@nuhs.edu.sg 

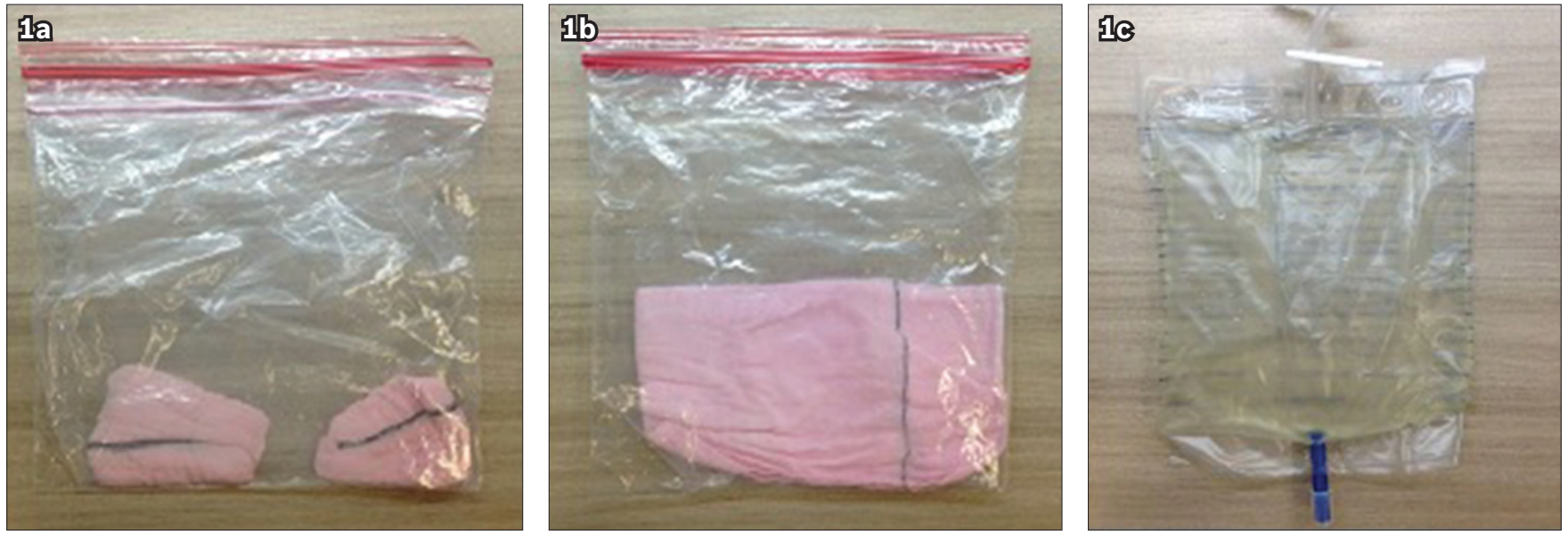

Fig. 1 Photographs show (a) Specimen 1 with $44 \mathrm{~mL}$ of fluid; (b) Specimen 2 with $144 \mathrm{~mL}$ of fluid; and (c) Specimen 3 with $288 \mathrm{~mL}$ of fluid.
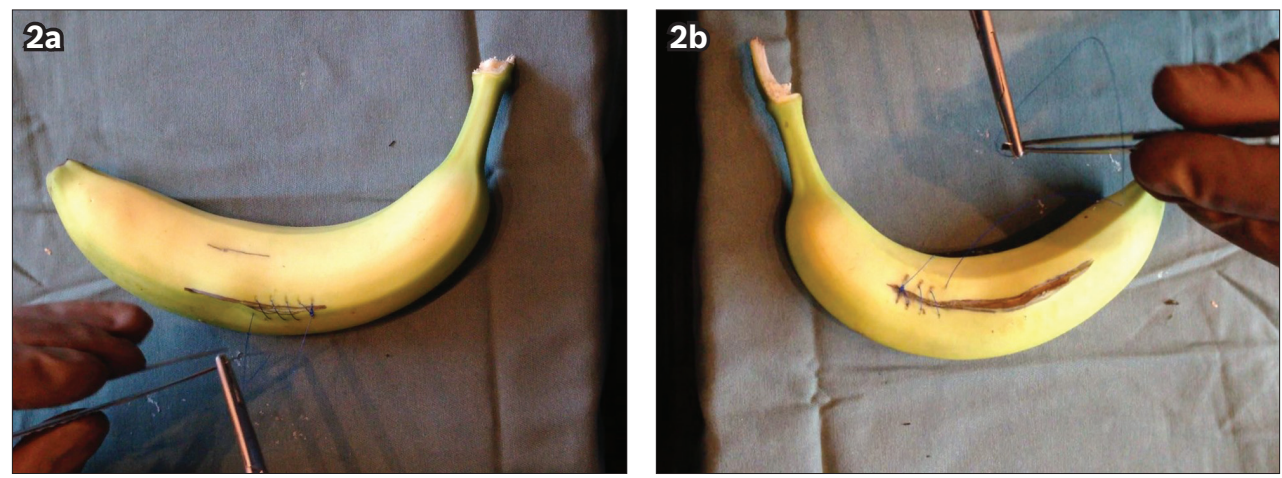

Fig. 2 Still capture of (a) Video 1 shows an incised banana being sutured; and (b) Video 2 shows an incised banana, with a visibly longer incision, being sutured.
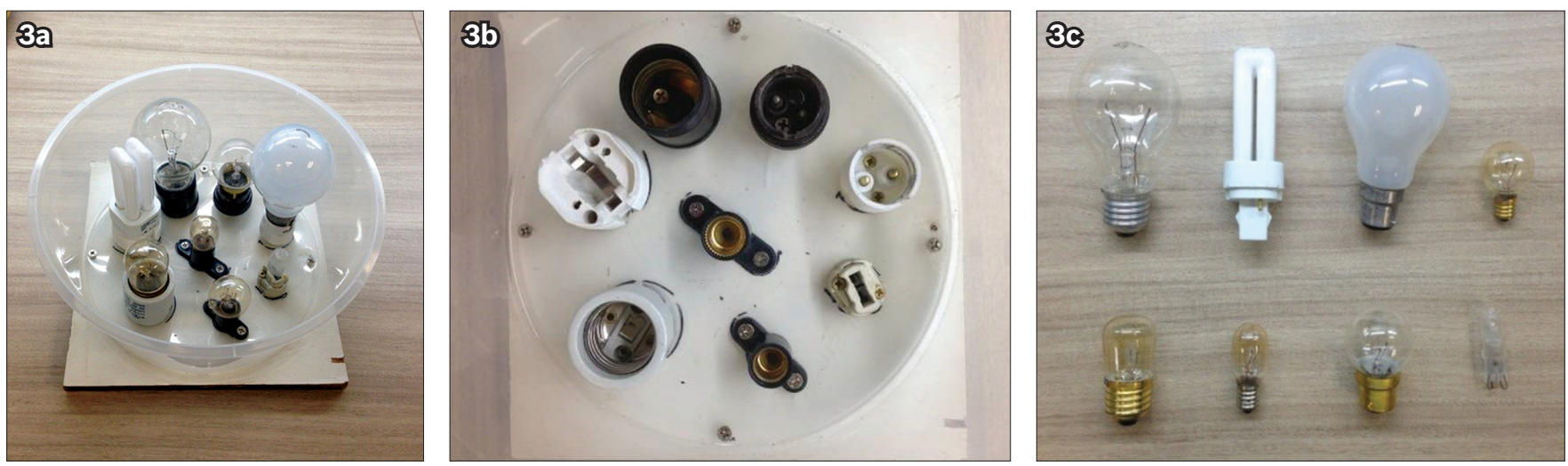

Fig. 3 Photographs show (a) light bulb test setup; (b) light sockets; and (c) light bulbs.

collection bag filled with $288 \mathrm{~mL}$ of iodine-tinged saline (Fig. 1). Participants were allowed to touch and handle the three bags for as long as they required to provide an estimate of the volume of fluid within each bag. These specimens were chosen because both surgeons and anaesthetists estimate fluid loss during surgery based on the weights of surgical swabs and sponges and the measure of intraoperative urine output in the catheter bag.

To test the participants' ability to estimate procedure duration, we prepared two videos of different durations (Fig. 2). These videos were shown to the participants via a smartphone. The first recorded procedure showed the suturing of an incised banana skin in a continuous fashion; the time taken to complete the procedure was 140 seconds. The video was played to completion and the participants had to estimate the time taken for the whole procedure based on what they had watched. The second recorded procedure showed the continuous suturing of a banana skin that had a longer incision; the time taken to complete the procedure was 170 seconds. Only a third of the video was shown to the participants, who, based on what they had watched (including the length of the incision), had to estimate the time it took for the whole incision to be sutured. Suturing of a banana skin was chosen as it represented skin closure in a surgical procedure. Based on our personal experience, this was the part of the surgery that anaesthetists were most likely to observe, as it would help them to reverse anaesthesia in a timely fashion.

To measure the participants' ability to change light bulbs, they were given a stopwatch and a plastic bowl on a wooden board 
with eight different light sockets attached to it (Fig. 3). The light bulbs lay randomly in the bowl. The participants were asked to find (and be in) a comfortable position and observe the setup without touching it, for as long as they wished. Once they were ready, the participants started the stopwatch. They then proceeded to work without removing any of the bulbs from the confines of the bowl. They had to attach the bulbs by matching them to the corresponding sockets, and then remove all the bulbs. When they were done, the participants stopped the stopwatch. The bowl in this setup simulated a lampshade. By having various light bulbs and sockets, we were able to test the participants' ability in not only attaching light bulbs, but also in choosing the correct bulb to match the socket.

Data was collected on a Microsoft Excel 2010 spreadsheet (Microsoft, Redmond, WA, USA) and analysed with IBM Statistical Package for the Social Sciences version 20 (IBM Corp, Armonk, NY, USA). Based on our hypothesis that it takes as many orthopaedic surgeons as anaesthetists to change a light bulb, the sample size was calculated on a type 1 error of 0.05 and a type 2 error of 0.2 . An a priorianalysis performed on the first 20 participants resulted in a mean \pm standard deviation of $75 \pm 18$ seconds. To detect a $15 \%$ difference in time to change light bulbs, we needed 31 participants in each group. Non-parametric data was analysed using the MannWhitney $U$ test. To calculate the mean percentage error for the groups, we first calculated the average percentage error (across all three volumes and both timings) for each participant. From this result, the mean percentage error for the group was obtained.

\section{RESULTS}

A total of 30 male anaesthetists and trainees (mean age 43 years, mean 15 years of experience) and 31 male orthopaedic surgeons and trainees (mean age 38 years, mean 10 years of experience) were recruited over the one-month study period (Table I). There were 14 orthopaedic consultants and 17 orthopaedic trainees, and 19 anaesthetic consultants and 11 anaesthetic trainees. There was a statistical difference between the two groups in terms of their age and experience. Three anaesthetists declined to participate in the study - two did not give reasons, while one gave the reason, "It's the only time I have the chance to say 'no' to doing a procedure requested by a surgeon." None of the orthopaedic surgeons approached declined to participate.

Orthopaedic surgeons underestimated the three volumes by $3.9 \%$, while anaesthetists overestimated the volumes by $5.1 \%$ (Table I). There was no statistically significant difference in estimation of fluid volumes between the orthopaedic surgeons and anaesthetists $(45.9 \mathrm{~mL}, 138.7 \mathrm{~mL}$ and $252.4 \mathrm{~mL}$ vs. $52.8 \mathrm{~mL}$, $153.5 \mathrm{~mL}$ and $255.0 \mathrm{~mL}, \mathrm{p}=0.925$ ) (Table II). The anaesthetists overestimated the duration of the two procedures by $21.2 \%$, while the orthopaedic surgeons overestimated the duration of the two procedures by $43.1 \%(p=0.066)$ (Table I). The orthopaedic surgeons overestimated the procedure duration more frequently than the anaesthetists ( 183.3 seconds and 263.9 seconds vs. 171.7 seconds and 203.5 seconds) (Table II). The anaesthetists had a faster mean time in changing light bulbs as compared to the orthopaedic surgeons (70.1 seconds vs. 74.1 seconds, $p=0.319$ ) (Table I).
Table I. Demographics and overall results of the participants $(n=61)$.

\begin{tabular}{lccc}
\hline Demographic & \multicolumn{2}{c}{ Mean \pm standard deviation } & p-value \\
\cline { 2 - 3 } & $\begin{array}{c}\text { Anaesthetists* } \\
(\mathbf{n}=\mathbf{3 0})\end{array}$ & $\begin{array}{c}\text { Orthopaedic } \\
\text { surgeons* }(\mathbf{n}=\mathbf{3 1})\end{array}$ & \\
\hline Age (yr) & $43.0 \pm 11.0$ & $37.6 \pm 10.0$ & 0.006 \\
Experience (yr) & $15.5 \pm 11.0$ & $9.5 \pm 9.5$ & 0.003 \\
Error in volume & $5.1 \pm 70.1$ & $-3.9 \pm 47.9$ & 0.925 \\
estimation (\%) & & & \\
Error in duration & $21.2 \pm 49.6$ & $43.1 \pm 56.3$ & 0.066 \\
estimation (\%) & & & 0.319 \\
Time taken to change & $70.1 \pm 16.5$ & $74.1 \pm 19.7$ & \\
light bulbs (s) & & & \\
\hline
\end{tabular}

*Includes trainees with a minimum of two years' experience in the specialty.

Table II. Volume and procedure duration estimates of the participants ( $n=61)$.

\begin{tabular}{lccc}
\hline Variable & \multicolumn{2}{c}{ Mean \pm standard deviation } & p-value \\
\cline { 2 - 3 } & $\begin{array}{c}\text { Anaesthetists* } \\
(\mathbf{n}=\mathbf{3 0})\end{array}$ & $\begin{array}{c}\text { Orthopaedic } \\
\text { surgeons* } \mathbf{( n = 3 1 )}\end{array}$ & \\
\hline $\begin{array}{l}\text { Volume estimate } \\
(\mathbf{m L})\end{array}$ & & & \\
$\quad$ Specimen 1 & $52.8 \pm 54.7$ & $45.9 \pm 32.6$ & 0.994 \\
Specimen 2 & $153.5 \pm 123.4$ & $138.7 \pm 101.6$ & 0.756 \\
Specimen 3 & $255.0 \pm 103.9$ & $252.4 \pm 56.0$ & 0.484 \\
Procedure duration & & & \\
estimate (s) & & & 0.199 \\
Video 1 & $171.7 \pm 72.4$ & $183.3 \pm 60.7$ & 0.067 \\
Video 2 & $203.5 \pm 89.0$ & $263.9 \pm 130.3$ & \\
\hline
\end{tabular}

Note: the actual volumes of Specimens 1,2 and 3 were $44 \mathrm{~mL}, 144 \mathrm{~mL}$ and $288 \mathrm{~mL}$, respectively; the actual procedure durations of Videos 1 and 2 were 140 seconds and 170 seconds, respectively. * Includes trainees with a minimum of two years' experience in the specialty.

\section{DISCUSSION}

The aim of the present study was to prove that there was no significant difference between orthopaedic surgeons and anaesthetists in their ability to estimate volumes, estimate procedure duration and complete the common task of changing bulbs. Accurate estimations of fluid losses during surgery and surgical procedure duration would allow optimal patient care and efficient utilisation of operating theatre resources. Furthermore, as both surgeons and anaesthetists have to review their postoperative patients, plan for the next day's operating schedule and perform non-clinical duties after completing their operating lists, the ability to complete the operating list on time would allow both groups to attend to other work commitments in a timely manner.

Volume estimation in the present study was surprisingly accurate in both groups, with a less than $5 \%$ error in estimation. Actual intraoperative fluid and blood loss is determined by measuring the amount of fluid in the suction canister and urine catheter, and by weighing surgical swabs. ${ }^{(5)}$ It is known that visual estimation is prone to error (overestimating at low volumes and underestimating at high volumes) ${ }^{(6)}$ In the case of paediatric spinal surgeries, Mooney et al concluded that orthopaedic surgeons had higher estimates for blood loss than anaesthetists. ${ }^{(7)}$

In the present study, both the orthopaedic surgeons and the anaesthetists were unable to accurately estimate procedure 
durations. In fact, the orthopaedic surgeons overestimated the procedure duration by $43.1 \%$, while the anaesthetists overestimated by $21.2 \%$. This result is unexpected, as it is popular belief that surgeons underestimate their surgical procedure duration and overestimate their operative ability. Although a study has concluded that surgeons provide significantly better estimates than computerised scheduling systems, there is still no consensus on whether the use of surgeons' estimates or historical surgical procedure durations are better in reducing the number of procedures that finish late. ${ }^{(8,9)}$ A very plausible explanation for our finding of gross overestimation of procedure duration (i.e. banana suturing) by both the orthopaedic surgeons and the anaesthetists is that an observer of a procedure tends to feel that the procedure is proceeding very slowly (be it a regional anaesthetic block or suturing of a wound). Future studies could be modified to improve on this element, by asking participants to estimate the time taken for them to perform a suturing task and actually perform the task subsequently.

In terms of the time taken to change the light bulbs, the result was largely similar between the two groups, proving that orthopaedic surgeons and anaesthetists were similarly capable at this task. A separate analysis performed, with age and experience as the denominator, did not reveal that younger persons were worse at changing light bulbs, contrary to our senior author's hypothesis. A potential source of error in the present study was the mismatch between the age and experience of the participants in the two groups. However, age and experience have not been proven to be associated with the ability to perform the three tasks evaluated in this study.

In a literature search that we conducted (in an attempt to account for any differences that may have occurred between the two groups), we found that orthopaedic surgeons have been portrayed as more aggressive and gorilla-like; they were also shown to enjoy golf as a sport. ${ }^{(10-12)}$ On the other hand, previous studies on the characteristics of anaesthetists reveal that they are more cooperative and harm-avoidant, and less novelty-seeking and reward-dependent. ${ }^{(13,14)}$ One correspondence article unveiled why anaesthetists have a special affinity for crossword puzzles. ${ }^{(15)}$

The strengths of the study include a prospective multicentre design and an adequate sample size. Also, the tests conducted were accurately and consistently replicated among all the participants. However, the present study was not without limitations. The testing material is novel and has not been proven to accurately correlate with the performance of the specialists in an actual clinical environment. Our light bulb setup also does not fully replicate the real-world process of changing light bulbs. The estimations of volume loss and procedure time could have been performed in an actual clinical environment to reflect the real-life scenario more accurately. We also had to exclude female participants so that any confounders due to gender differences could be eliminated (neither were there sufficient women in the sample for a meaningful analysis). The random nature of participant invitation meant that not all potentially eligible participants from all three institutions were included in the study. The difference in the mean age of the two groups may also have been a potential confounder (a group of younger orthopaedic surgeons were compared with a group of older anaesthetists). One possible explanation for this age difference is that anaesthetists have a longer career lifespan, and being less reward-dependent, there may have been more likely to continue working in one of the three public hospitals that were involved in this study.

To conclude, in a testing environment, orthopaedic surgeons are as good as anaesthetists in: (a) estimating fluid volumes in commonly seen surgical specimens; (b) estimating the time taken to complete a commonly seen surgical procedure; and (c) changing light bulbs. An orthopaedic surgeon's estimate of fluid loss during surgery should not be discounted by their anaesthetist colleagues. Both groups of specialists are poor at estimating procedure duration. The findings of the present study may form the basis for future clinical studies, which may have results that can be extrapolated to an actual clinical environment. And to answer the question of how many orthopaedic surgeons it takes to change a light bulb, the answer is that it takes as many orthopaedic surgeons as it does anaesthetists to change a light bulb (although they may be occupied with golf or crossword puzzles).

\section{REFERENCES}

1. Kay P. Orthopaedic Joke Section [online]. Available at: mrkay.co.uk/joke. htm. Accessed September 19, 2013.

2. Subramanian P, Kantharuban S, Subramanian V, Willis-Owen SA, WillisOwen CA. Orthopaedic surgeons: as strong as an ox and almost twice as clever? Multicentre prospective comparative study. BMJ 2011; 343:d7506.

3. World Health Organization. World Alliance for Patient Safety. Global Patient Safety Challenges. The First Challenge: Clean Care is Safer Care. The Second Challenge: Safe Surgery Saves Lives. A WHO Initiative. Supported by The Association of Surgeons of India. Available at: www.who.int/ patientsafety/safesurgery/tools_resources/SSSL_Checklist_finalJun08.pdf. Accessed September 19, 2013

4. Chen Y, Tai BC, Nayak D, et al. Blood loss in spinal tumour surgery and surgery for metastatic spinal disease: a meta-analysis. Bone Joint J 2013; 95-B:683-8.

5. Lee $M H$, Ingvertsen $B T$, Kirpensteijn J, Jensen $A L$, Kristensen AT. Quantification of surgical blood loss. Vet Surg 2006; 35:388-93.

6. Dildy GA 3rd, Paine AR, George NC, Velasco C. Estimating blood loss: can teaching significantly improve visual estimation? Obstet Gynecol 2004; 104:601-6.

7. Mooney JF, Barfield WR. Validity of Estimates of Intraoperative Blood Loss in Pediatric Spinal Deformity Surgery. Spine Deformity 2013; 1:21-4.

8. Wright IH, Kooperberg C, Bonar BA, Bashein G. Statistical modeling to predict elective surgery time. Comparison with a computer scheduling system and surgeon-provided estimates. Anesthesiology 1996; 85:1235-45.

9. Zhou J, Dexter F, Macario A, Lubarsky DA. Relying solely on historical surgical times to estimate accurately future surgical times is unlikely to reduce the average length of time cases finish late. J Clin Anesth 1999; 11:601-5.

10. Barlow T, Wight A, Barlow D. A cross-sectional study of aggression levels in physicians and orthopaedic surgeons: impact on specialty selection and training? JRSM Short Rep 2012; 3:83.

11. Fox JS, Bell GR, Sweeney PJ. Are orthopaedic surgeons really gorillas? BMJ 1990; 301:1425-6.

12. Maritz NGJ, Kreuser I, Majake M, Masinga N. A comparison of character traits of orthopaedic surgeons and a mixed control group of general surgeons and physicians. J Bone Joint Surg Br 2005; 87-B Suppl 3:276.

13. Kluger MT, Laidlaw TM, Kruger N, Harrison MJ. Personality traits of anaesthetists and physicians: an evaluation using the Cloninger Temperament and Character Inventory (TCl-125). Anaesthesia 1999; 54:926-35.

14. Adudu OP. What impressions do medical students of the University of Benin have about anaesthesia and anaesthesiologists? Niger Postgrad Med J 2005; 12:250-4.

15. McCormick WO. Anaesthetists and Crossword Puzzles. Br Med J 1968; $3: 679$ 